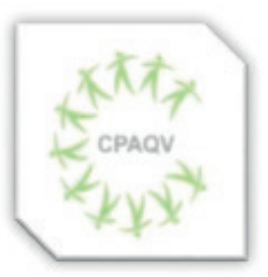

ISSN: $2178-7514$

Vol. 13 | No. 3| Ano 2021
ARTIGO DE REVISÃO

\section{A PRÁTICA REGULAR DE EXERCÍCIO FÍSICO NO CONTROLE DA HIPERTENSÃO ARTERIAL}

\section{The regular practice of physical exercise in the control of arterial hypertension}

\begin{abstract}
RESUMO
O objetivo deste estudo foi revisar e analisar os benefícios da prática do exercício físico de forma regular para pessoas com hipertensão arterial. Métodos: O presente estudo foi realizado a partir de uma revisão narrativa envolvendo buscas nas bases de dados SciELO e Google Acadêmico, no idioma português com as palavras chave: prática regular de exercício físico, controle da hipertensẫo arterial. Resultados: Foram identificados impactos positivos da prática regular de exercício físico na melhora de variáveis relacionadas a hipertensão arterial sistêmica e controle de doenças, alêm de auxiliar na manutenção do peso corporal, melhorar o sono e a qualidade de vida. $O$ volume de treino em minutos (duração da sessão [min] x frequência semanal x duração do treino em semanas) tem associação moderada com a redução percentual da pressão arterial sistólica $(\mathrm{r}=0,53 ; \mathrm{p}<0,05)$ e baixa com a redução percentual da pressão arterial diastólica $(\mathrm{r}=0,33 ; \mathrm{p}<0,05)$. Conclusão: O exercício físico, principalmente quando praticado de forma regular, tem papel essencial não somente no tratamento não medicamentoso para controle da hipertensão arterial, mas também na manutenção da qualidade de vida dos indivíduos. $\mathrm{O}$ volume de treino é uma variável influente na magnitude das reduções dos indicadores de hipertensão.
\end{abstract}

Palavras-chave: Atividade Física. Doença Crônica. Obesidade. Sedentarismo. Pressão Arterial.

\begin{abstract}
Thus, the aim of this study was to review and to analyses the benefits of physical exercise on a regular basis for people with arterial hypertension. Methods: The present study was carried out from a narrative review involving searches in the SciELO and Google Scholar databases, in Portuguese with the keywords: regular practice of physical exercise, control of arterial hypertension. Results: Positive impacts of regular physical exercise were identified to improve variables related to systemic arterial hypertension and disease control, in addition to maintaining body weight, improving sleep and quality of life. The training volume in minutes (session duration [min] $\mathrm{x}$ weekly frequency $\mathrm{x}$ training duration in weeks) has a moderate association with the percentage reduction in systolic blood pressure $(r=0.53 ; \mathrm{p}<0.05)$ and low with the percentage reduction in diastolic blood pressure $(r=0.33 ; \mathrm{p}<0.05)$. Conclusion: Physical exercise, especially when practiced on a regular basis, plays an essential role not only in non-drug treatment for the control of arterial hypertension, but also in maintaining the quality of life of individuals. Training volume is an influential variable in the magnitude of reductions in hypertension indicators.
\end{abstract}

Keywords: Physical Activity. Chronic Disease, Obesity. Sedentary Lifestyle. Blood Pressure.

Autor de correspondência

Prof. Dr. Anderson dos Santos Carvalho

E-mail: anderson.carvalho@docente.unip.br

E-mail: ander_uai@hotmail.com

DOI: $10.36692 / \mathrm{v} 13 \mathrm{n} 3-05 \mathrm{R}$ 


\section{INTRODUÇÃO}

Atualmente, grande parte da população Brasileira permanece num estilo de vida sedentário, com predisposição a um alto nível de estresse e de forma frequente consumindo alimentos do tipo fast food, que são pobres em nutrientes para o adequado funcionamento do corpo humano. O consumo frequente desses alimentos ultra processados favorece o maior consumo calórico e aumento do peso corporal, que pode evoluir para sobrepeso e obesidade ${ }^{(1)}$. Assim, a população tem apresentado aumento de doenças crônicas degenerativas como obesidade, diabetes mellitus tipo 2, câncer, insuficiência cardíaca e hipertensão $\operatorname{arterial}^{(2)}$.

A hipertensão arterial sistêmica (HAS) é uma doença cônica não transmissível de diagnóstico e identificação frequente pelos médicos e profissionais de saúde (3). É caracterizada pelo aumento da demanda de trabalho do músculo cardíaco para manter a perfusão sanguínea adequada para os outros órgãos, em razão da maior resistência oferecida pelas artérias. Segundo dados do Ministério da Saúde, a hipertensão arterial acomete $25 \%$ dos adultos no Brasil, e está relacionada diretamente com o sedentarismo e má alimentação ${ }^{(4)}$. As recomendações para o tratamento da HAS em níveis iniciais é de se iniciar pelas modificações dos hábitos de vida, como alimentação com menor teor de sódio e adoção de dietas específicas, a exemplo da DASH (Dietary Approaches to Stop Hypertension), caracterizada pelo maior consumo de hortaliças, leguminosas, grãos integrais e frutas, alimentos com baixo teor de gordura saturada e trans e colesterol, com elevada quantidade de gordura monoinsaturada (azeite de oliva) e ácidos graxos ômega-3, quantidades adequadas de potássio, magnésio e cálcio $^{(5)}$. Ainda em níveis iniciais, a HAS pode ser controlada com práticas regulares de exercício físico e aumento da prática diária de atividade física (AF) não sistematizada (2, 6). Assim, somente quando a estratégia de tratamento não farmacológico for insuficiente para controlar a pressão arterial é que deveria ser iniciado o tratamento farmacológico. Recomenda-se que os indivíduos hipertensos iniciem programas de exercício físico regular, desde que submetidos à avaliação clínica prévia e sob supervisão de um profissional de Educação Física. A pratica de atividade física tem um efeito sobre o sistema autônomo, modificando o sistema cardiovascular a partir da regulação dos sistemas simpáticos e parassimpático ${ }^{(7)}$. Esses sistemas controlam a frequência cardíaca, a força contráctil e o redirecionamento do fluxo sanguíneo para zonas com maior necessidade vascular, resultando em um melhor controle da pressão $\operatorname{arterial}^{(7)}$.

Os exercícios aeróbios e anaeróbios são sabidamente benéficos ao hipertenso ${ }^{(8)}$ tendo influência significativa na utilização de dosagem de alguns medicamentos anti-hipertensivos ${ }^{(9)}$. 
No entanto, esforços para controlar a pressão arterial têm sido em geral concentrados exclusivamente na terapia farmacológica, sabidamente de comprovada eficácia no controle da pressão arterial com consequente redução de suas comorbidades ${ }^{(10)}$. É evidente que os medicamentos são necessários para alguns casos, no entanto, podem apresentar diversos efeitos colaterais, como, alteração na frequência cardíaca, tontura, retenção liquida, dor de cabeça, impotência, náuseas e sudorese ${ }^{(11)}$. Por isso, tanto quanto possível, outras estratégias alternativas não devem ser excluídas, como o exercício físico ${ }^{(10)}$. Este tem apresentado um bom custo-efetividade em razão de sua ampla aplicabilidade e influência na qualidade de vida. Entretanto, existe considerável lacuna sobre o tratamento não farmacológico com ênfase no exercício físico, em hipertensos medicados e não medicados, algo que, apesar de ser fortemente recomendado pelas diretrizes, é muito negligenciado na prática clínica $^{(10,12)}$. É notório que existe resistência por parte da população em iniciar a prática de exercícios físicos, e a maioria prefere caminho "de menor esforço" e não leva em consideração que a prática de exercício físico pode controlar a pressão arterial e promover outros benefícios: controle do peso corporal, autoestima, melhora no sono e aumento da expectativa de $\mathrm{vida}^{(2)}$. Está evidente na literatura a importância da prática do exercício físico para a saúde e bemestar $(2,6,8)$, porém, é preciso apresentar seus benefícios e incentivar os sujeitos com doenças crônicas não transmissíveis para praticarem, especialmente os hipertensos, que têm boa possibilidade de controle da doença. Em alguns casos é possível suspender o uso de fármacos, gerando de forma direta e indireta, economia para o usuário e para o país. Assim sendo, o objetivo deste estudo é apresentar os benefícios da prática do exercício físico de forma regular para pessoas com hipertensão arterial.

\section{MÉTODOS}

Trata-se de um estudo de revisão da literatura. As bases de dados utilizadas foram SciELO e Google Acadêmico. O período de publicação dos artigos selecionados foi de 1995 a 2020. Os descritores utilizados foram prática regular exercício físico e controle da hipertensão arterial. Foram incluídos: a) estudos clínicos com delineamento transversal, longitudinal, de caso controle e aleatorizados, além de estudos de revisão relacionados à exercício físico e hipertensão arterial; b) estudos publicados somente no idioma português (tanto as bases de dados como o idioma foram definidos com base no objeto de estudo). Foram excluídos artigos com informações incompletas que não atenderam aos critérios de elegibilidade.

A princípio os artigos foram eleitos de acordo com os critérios descritos acima. Esta revisão compreendeu três fases. $\mathrm{Na}$ primeira foi realizada a pesquisa nas bases de dados. $\mathrm{Na}$ 
segunda fase, após a seleção, foram realizadas análises dos títulos e resumos para determinar a elegibilidade dos estudos. Na terceira e última fase foram avaliados os textos completos. Foram realizadas análises críticas de conteúdo, considerando o mérito científico de cada estudo e possíveis relações de similaridades ou conflitos entre eles. Este processo foi realizado por dois pesquisadores de forma independente.

Após a identificação dos principais resultados dos estudos selecionados, foi verificada a relação (correlação de Pearson e gráficos de dispersão) entre o volume de treinamento com a redução percentual da
Pressão Arterial Sistólica (PAS) e Diastólica (PAD). O volume de treinamento foi calculado com a equação:

Volume de treinamento $[\mathrm{min}]=$ duração da sessão[min] x frequência semanal x duração do treinamento $\left[\mathrm{n}^{\circ}\right.$ de semanas]

\section{RESULTADOS E DISCUSSÃO}

Os temas abordados nesta revisão narrativa foram definidos a partir de 23 artigos científicos selecionados de acordo com os critérios de inclusão descrito nos métodos (Quadro 1).

Quadro 1. Artigos selecionados para construção desta revisão narrativa.

\begin{tabular}{|c|c|c|c|}
\hline Autores & Ano & Título & Objetivo \\
\hline Tanaka et al. ${ }^{(13)}$ & 1997 & $\begin{array}{l}\text { Swimming training lowers the resting blood } \\
\text { pressure in individuals with hypertension }\end{array}$ & $\begin{array}{l}\text { Determinar os efeitos do treinamento de natação no sangue arterial } \\
\text { em repouso pressão em pacientes hipertensos estágios } 1 \text { e } 2 \text {. } \\
\text { Adicionalmente, examinar várias variáveis mecanicistas que poderia } \\
\text { explicar as mudanças induzidas pelo treinamento, se houver } \\
\text { observada, na pressão arterial. }\end{array}$ \\
\hline Arca et al. (14) & 2004 & $\begin{array}{l}\text { Efeitos da hidrocinesioterapia na pressão } \\
\text { arterial e nas medidas antropométricas em um } \\
\text { grupo de mulheres hipertensas }\end{array}$ & $\begin{array}{l}\text { Verificar os efeitos de um programa de hidrocinesioterapia na } \\
\text { pressão arterial e nas medidas antropométricas em mulheres } \\
\text { hipertensas. }\end{array}$ \\
\hline $\begin{array}{l}\text { Ciolac, } \\
\text { Guimarães. (15) }\end{array}$ & 2004 & Exercício físico e síndrome metabólica & $\begin{array}{l}\text { Demonstrar o papel da prática regular de atividade física na } \\
\text { prevenção e tratamento da síndrome metabólica, bem como } \\
\text { descrever a quantidade e modalidade de exercício necessário para } \\
\text { esse fim. }\end{array}$ \\
\hline Cox et al. ${ }^{(16)}$ & 2006 & $\begin{array}{l}\text { Blood pressure rise with swimming versus } \\
\text { walking in older women: the Sedentary Women } \\
\text { Exercise Adherence Trial } 2 \text { (SWEAT 2) }\end{array}$ & $\begin{array}{l}\text { Hipotetizar que um período de } 6 \text { meses programa supervisionado de } \\
\text { caminhada de intensidade moderada em mulheres mais velhas } \\
\text { normotensas / controladas não obesas iriam resultar em uma maior } \\
\text { redução na PA de repouso em comparação com um programa de } \\
\text { natação de intensidade semelhante. }\end{array}$ \\
\hline Simões et al. .17) $^{(17)}$ & 2007 & $\begin{array}{l}\text { Efeitos do treinamento de hidroginástica na } \\
\text { aptidão cardiorrespiratória e nas variáveis } \\
\text { hemodinâmicas de mulheres hipertensas }\end{array}$ & $\begin{array}{l}\text { Avaliar os efeitos de um programa de treinamento aeróbio de } \\
\text { hidroginástica sobre a aptidâo cardiorrespiratória e sobre variáveis } \\
\text { hemodinâmicas de mulheres hipertensas praticantes de exercícios } \\
\text { fisicos no Centro de Qualidade de Vida da Universidade Metodista } \\
\text { de Piracicaba (CQV-UNIMEP). }\end{array}$ \\
\hline Piazza et al. ${ }^{(18)}$ & 2008 & $\begin{array}{l}\text { Efeitos de exercícios aquáticos sobre a aptidão } \\
\text { cardiorrespiratória e a pressão arterial em } \\
\text { hipertensas }\end{array}$ & $\begin{array}{l}\text { Avaliar o efeito de um programa de exercícios } \\
\text { hidrocinesioterapêuticos sobre a capacidade funcional aeróbia e a } \\
\text { pressão arterial (PA) em mulheres hipertensas. }\end{array}$ \\
\hline $\begin{array}{l}\text { Gimenes et al. } \\
\text { (19) }\end{array}$ & 2008 & $\begin{array}{l}\text { Impacto da Fisioterapia Aquática na Pressão } \\
\text { Arterial de Idosos }\end{array}$ & $\begin{array}{l}\text { Avaliar e comparar a variação da pressão arterial em indivíduos } \\
\text { idosos que praticam a fisioterapia aquática em grupo em dois } \\
\text { periodos definidos (antes e após intervenção). }\end{array}$ \\
\hline Chen et al. ${ }^{(20)}$ & 2010 & $\begin{array}{l}\text { Effects of one-year swimming training on blood } \\
\text { pressure and insulin sensitivity in mild } \\
\text { hypertensive young patients }\end{array}$ & $\begin{array}{l}\text { Avaliar o efeito do treinamento de nataçâo de um ano sobre a PA } \\
\text { com a inclusão de } 7 \text { leves pacientes hipertensos e } 16 \text { normotensos } \\
\text { de mesma idade. }\end{array}$ \\
\hline
\end{tabular}


A prática regular de exercício físico no controle da hipertensão arterial

\begin{tabular}{|c|c|c|c|}
\hline $\begin{array}{l}\text { Guedes, } \\
\text { Lopes. }\end{array}$ & 2010 & $\begin{array}{l}\text { Exercício físico em portadores de hipertensão } \\
\text { arterial: uma análise conceitual }\end{array}$ & $\begin{array}{l}\text { Analisar o conceito de exercício fisico em portadores de hipertensão } \\
\text { arterial, identificando os possiveis atributos críticos e os fatores } \\
\text { antecedentes e consequentes. }\end{array}$ \\
\hline $\begin{array}{l}\text { Farahani et } \\
\text { al.(22) }\end{array}$ & 2010 & $\begin{array}{l}\text { The effects of a 10-weekwater aerobic exercise } \\
\text { on the resting blood pressure in patients with } \\
\text { essential hypertension }\end{array}$ & $\begin{array}{l}\text { Investigar os efeitos do treinamento aeróbio aquático sobre a } \\
\text { pressão arterial em pacientes hipertensos com hipertensão estágio } \\
1 \text { ou } 2 \text { referenciados às Clínicas da Universidade de Teerã. }\end{array}$ \\
\hline $\begin{array}{l}\text { Nualmin et } \\
\text { al. }{ }^{(23)}\end{array}$ & \begin{tabular}{|l|}
2012 \\
\end{tabular} & $\begin{array}{l}\text { fects of swimming training on blood pressure } \\
\text { Id vascular function in adults }>50 \text { years of age }\end{array}$ & $\begin{array}{l}\text { Determinar o efeito da intervenção do exercício de natação na PA } \\
\text { mediçôes das funções vasculares em adultos com } 50 \text { anos de idade } \\
\text { com aumento da PA. }\end{array}$ \\
\hline & 2013 & $\begin{array}{l}\text { xercício fisico controla pressão arterial e } \\
\text { elhora qualidade de vida. }\end{array}$ & $\begin{array}{l}\text { Avaliar o efeito do tratamento exclusivo com exercício físico na } \\
\text { pressão arterial e qualidade de vida de hipertensos. }\end{array}$ \\
\hline $\begin{array}{l}\text { Matavelli, et al. } \\
\text { (9) }\end{array}$ & 014 & $\begin{array}{l}\text { Hipertensão arterial sistêmica e a prática regular } \\
\text { de exercícios físicos como forma de controle: } \\
\text { Revisão de Literatura. }\end{array}$ & $\begin{array}{l}\text { A presente revisão faz uma abordagem sobre os aspectos clínicos } \\
\text { da hipertensão arterial sistólica e o potencial da prática de exercícios } \\
\text { físicos regulares como método não farmacológico no seu controle. }\end{array}$ \\
\hline $\begin{array}{l}\text { Santos, Costa, } \\
\text { Kruel.(24) }\end{array}$ & 2014 & $\begin{array}{l}\text { Efeitos de exercícios aeróbicos aquáticos sobre } \\
\text { a pressão arterial em adultos hipertensos: } \\
\text { revisão sistemática. }\end{array}$ & $\begin{array}{l}\text { Revisar sistematicamente os efeitos agudos e crônicos de } \\
\text { exercícios aeróbicos aquáticos sobre a pressão arterial de } \\
\text { indivíduos hipertensos. }\end{array}$ \\
\hline $\begin{array}{l}\text { Zaar, Reis, } \\
\text { Sbardelotto }\end{array}$ & 2014 & $\begin{array}{l}\text { Efeitos de um programa de exercícios fisicos } \\
\text { sobre a pressão arterial e medidas } \\
\text { antropométricas. }\end{array}$ & $\begin{array}{l}\text { Analisar os efeitos de um programa de condicionamento físico } \\
\text { realizado por um periodo de } 24 \text { meses sobre pressão arterial } \\
\text { composiçấo corporal em individuos normotensos e pré-hipertensos. }\end{array}$ \\
\hline es & 014 & $\begin{array}{l}\text { Heated water-based exercise training reduces } \\
\text { 24-hour ambulatory blood pressure levels in } \\
\text { resistent hypertensive patients: A randomized } \\
\text { controlled trial (HEx trial) }\end{array}$ & $\begin{array}{l}\text { físico à base de água aquecida } \\
\text { tes. }\end{array}$ \\
\hline Felício et al. ${ }^{(27)}$ & \begin{tabular}{|l|}
2017 \\
\end{tabular} & $\begin{array}{l}\text { Atividade física no equilíbrio metabólico da } \\
\text { hipertensão arterial }\end{array}$ & $\begin{array}{l}\text { Avaliar o controle da HAS em resposta a um programa de exercícios } \\
\text { fisicos e de alguns fatores que são considerados como risco porque } \\
\text { comprometem a expectativa de vida da população por causarem } \\
\text { eventos cardiovasculares responsáveis por incapacidade precoce, } \\
\text { invalidez e óbito. }\end{array}$ \\
\hline (28) & \begin{tabular}{|l|}
2017 \\
\end{tabular} & $\begin{array}{l}\text { Efeitos de um programa de exercício físico ao ar } \\
\text { livre em diabéticos e hipertensos atendidos em } \\
\text { um núcleo de apoio à saúde da família de lagoa } \\
\text { da prata-MG }\end{array}$ & $\begin{array}{l}\text { Avaliar os efeitos de um programa sistematizado de exercício físico } \\
\text { em adultos e idosos diabéticos e/ou hipertensos atendidos em um } \\
\text { Núcleo de Apoio à Saúde da Família (NASF) da cidade de Lagoa da } \\
\text { Prata-MG. }\end{array}$ \\
\hline $\begin{array}{l}\text { Horte } \\
(29)\end{array}$ & 018 & $\begin{array}{l}\text { Efeito de exercícios físicos sobre fatores de } \\
\text { risco cardiovascular em idosos hipertensos. }\end{array}$ & $\begin{array}{l}\text { Avaliar os efeitos de um programa de exercícios na redução dos } \\
\text { fatores de risco cardiovascular em idosos sedentários e } \\
\text { hipertensos. }\end{array}$ \\
\hline $\begin{array}{l}\text { Damá } \\
(30)\end{array}$ & 2019 & $\begin{array}{l}\text { Os efeitos de um programa de atividade física } \\
\text { na pressão arterial e frequência cardiaca de } \\
\text { pacientes hipertensos e deficientes físicos. }\end{array}$ & $\begin{array}{l}\text { Investigar os efeitos de um programa de atividade física, com } \\
\text { intensidade moderada, na pressão arterial e frequência cardiaca dos } \\
\text { pacientes com deficiência física e hipertensão arterial em um } \\
\text { município do interior de Minas Gerais/Brasil. }\end{array}$ \\
\hline $\operatorname{Lima}^{(31)}$ & \begin{tabular}{|l|}
2019 \\
\end{tabular} & $\begin{array}{l}\text { Análise dos efeitos do exercício físico aplicado } \\
\text { aos pacientes com hipertensão arterial } \\
\text { sistêmica e diabetes de mellitus assistidos pela } \\
\text { equipe de estratégia saúde e família da cidade } \\
\text { de Paracatu-MG }\end{array}$ & $\begin{array}{l}\text { Avaliar os efeitos do exercício físico em pacientes portadores de } \\
\text { diabetes e/ ou hipertensão assistidos pelo ESF- Santana. }\end{array}$ \\
\hline $\begin{array}{l}\text { Cassi } \\
\text { (32) }\end{array}$ & 20 & $\begin{array}{l}\text { Efeitos do exercício físico sobre o risco } \\
\text { cardiovascular e qualidade de vida em idosos } \\
\text { hipertensos }\end{array}$ & $\begin{array}{l}\text { feitos de um protocolo misto de exercícios físicos sobre o } \\
\text { rdiovascular (RCV), qualidade de vida e presença de } \\
\text { depressivos em idosos hipertensos. }\end{array}$ \\
\hline Vargas & \begin{tabular}{|l|}
2020 \\
\end{tabular} & $\begin{array}{l}\text { Efeito agudo dos exercícios resistidos e } \\
\text { exercícios aeróbicos sobre a pressão arterial de } \\
\text { homens hipertensos leves. }\end{array}$ & $\begin{array}{l}\text { Analisar o efeito agudo de exercícios resistidos e aeróbicos sobre a } \\
\text { pressão arterial em homens hipertensos leves. }\end{array}$ \\
\hline
\end{tabular}

Sabe-se que a prática regular de exercício físico traz numerosos benefícios para o corpo humano ${ }^{(2)}$, entre eles, o controle ou até a reversão de um quadro de hipertensão(33). Estudos epidemiológicos e clínicos têm demonstrado efeitos benéficos da prática de atividade física sobre a pressão arterial em indivíduos de todas as idades ${ }^{(32)}$. Alto nível de atividade física diária está associado a níveis de pressão arterial em repouso mais bem controlados em hipertensos ${ }^{(2)}$, pois ocorre aumento da sensibilidade no controle barorreflexo, cardiopulmonar e quimiorreflexo central e periférico, o que melhora o sistema parassimpático $^{(34)}$. A prática regular de exercício físico tem demonstrado prevenir o aumento da pressão arterial associado à idade, mesmo em indivíduos com risco aumentado de desenvolvê-la $a^{(2,15)}$. Os fatores que influenciam no desenvolvimento da hipertensão arterial vão desde hereditariedade, envelhecimento até obesidade, sedentarismo e estresse. O 
exercício físico pode modular positivamente esses fatores ${ }^{(29)}$. Por exemplo, a prática de exercício físico estimula a liberação de hormônios, como a adrenalina, responsável pela ativação do sistema fisiológico, e após cessar os movimentos ocorre a liberação do neurotransmissor de acetilcolina e endorfina, as quais são responsáveis pela sensação de bemestar e relaxamento do organismo, atuando também na diminuição do estresse.

Um estudo feito com 16 voluntários normotensos, sendo sete mulheres e nove homens e 19 voluntários pré-hipertensos, sendo 8 mulheres e 11 homens, baseado em um programa de condicionamento físico de 24 meses de duração, realizado três vezes por semana com 60 minutos por sessão em intensidade de 50-80\% da FCsub-máxima, resultou na redução da PAS e PAD em ambos os grupos, justamente com redução no perímetro da cintura e da adiposidade central, além do aumento significativo na massa magra ${ }^{(25)}$.

Parte da população normalmente procura auxílio médico apenas quando inicia o aparecimento dos sintomas e, portanto, a hipertensão está em grau avançado. Por conta disso, as modificações necessárias para melhora podem ser vistas como extremas pelo indivíduo, incluindo a mudança dos hábitos alimentares, início da prática de exercício físico, redução/ cessação do hábito de fumar para os tabagistas e outras recomendações específicas.
As estratégias que podem ser utilizadas para o desenvolvimento de metas junto a portadores de hipertensão arterial a incluem a educação em saúde enfatizando os benefícios, os caminhos para superar as dificuldades, o suporte familiar e de amigos, a motivação para a prática, o uso do pedômetro e a determinação do plano para o exercício e metas a serem atingidas $^{(21)}$. É papel importante do profissional da saúde ou da equipe multidisciplinar mostrar aos indivíduos os benefícios da prática de exercício físico, porém, a maioria deles resiste devido aos péssimos hábitos aos quais estiveram acostumados durante grande parte da vida ${ }^{(28)}$. Enfatiza-se que um longo período de aconselhamento pode ser necessário para motivação e mudança de comportamento. A possibilidade de intervenções motivacionais de enfermagem que estimulem a superação da resistência ao exercício são questões fundamentais na tentativa de iniciar e manter uma prática regular de exercício físico. A falta de motivação e ausência de interesse pelo exercício físico são elementos importantes que influenciam, de forma direta, tanto a adesão, quanto a manutenção em programas de exercício físico. Nesse sentido, grupos de apoio são fundamentais para apoiar essa prática ${ }^{(2)}$.

O Quadro 2 retrata a relação entre variáveis do treinamento físico (tipo, duração, frequência e o tempo das sessões, intensidade e frequência de participação) com a alteração (\%) dos parâmetros de HAS. 
A prática regular de exercício físico no controle da hipertensão arterial

Quadro 2. Relação entre os parâmetros do treinamento físico com a alteração percentual em indicadores de hipertensão arterial sistêmica.

\begin{tabular}{|c|c|c|c|c|c|c|c|c|c|c|}
\hline \multirow[b]{2}{*}{ Autor } & \multirow{2}{*}{ Tipo } & \multirow{2}{*}{$\begin{array}{l}\text { Duraçäo } \\
\text { (meses) }\end{array}$} & \multirow{2}{*}{$\begin{array}{c}\text { Frequência } \\
\text { semanal }\end{array}$} & \multirow{2}{*}{$\begin{array}{c}\text { Duraçäo da } \\
\text { Sessão } \\
\text { (minutos) }\end{array}$} & \multirow[b]{2}{*}{ Intensidade } & \multirow{2}{*}{$\begin{array}{l}\text { Frequência de } \\
\text { Participação }\end{array}$} & \multicolumn{4}{|c|}{ Alteração (\%) } \\
\hline & & & & & & & PAS & PAD & FCrep & Duplo produto \\
\hline Tanaka et al. ${ }^{(13)}$ & Natação & 2,5 & 3 & 60 & $60 \% V_{2} \max$ & $\begin{array}{l}\text { Nẫo há detalhamento } \\
\text { (nenhum voluntário foi } \\
\text { excluído) }\end{array}$ & \begin{tabular}{|l|} 
Grupo \\
intervenção: \\
$\downarrow 4$ \\
Grupo \\
controle: $\downarrow 7,1$ \\
\end{tabular} & \begin{tabular}{|l} 
Grupo intervençăo: \\
$\downarrow 2,1$ \\
Grupo controle: $s /$ \\
alteraçấo \\
\end{tabular} & $\begin{array}{l}\text { Grupo intervençäo: } \\
\downarrow 12,5 \\
\text { Grupo controle: } \\
\downarrow 2,3 \\
\end{array}$ & $\begin{array}{l}\text { Grupo intervenção: } \\
\downarrow 16 \\
\text { Grupo controle: } \downarrow 3\end{array}$ \\
\hline Arca et al. ${ }^{(14)}$ & Hidroginástica & 2,5 & 2 & 60 & $60 \% \mathrm{FC}_{\mathrm{max}}$ & $\begin{array}{l}\text { Permitido 20\% de } \\
\text { faltas }\end{array}$ & $\downarrow 3,6$ & $\downarrow 11,1$ & \begin{tabular}{|l|} 
Năo há \\
detalhamento
\end{tabular} & $\begin{array}{l}\text { Nẫo há } \\
\text { detalhamento }\end{array}$ \\
\hline Cox et al. ${ }^{(16)}$ & $\begin{array}{l}\text { Nataçẫo + } \\
\text { caminhada }\end{array}$ & 6 & $\begin{array}{l}\text { Năo há } \\
\text { detalhamen } \\
\text { to }\end{array}$ & $\begin{array}{l}\text { Natação: } 60 \\
\text { Caminhada: } \\
\text { 30 }\end{array}$ & $60-70 \% \mathrm{FC}_{\text {max }}$ & $\begin{array}{l}13,8 \% \text { nấo } \\
\text { permaneceram no } \\
\text { programa }\end{array}$ & $\begin{array}{l}\text { Natação: } s / \\
\text { alteracăo } \\
\text { Caminhada: } \\
\uparrow 1,2\end{array}$ & $\begin{array}{l}\text { Natacẫo: } \downarrow 10,5 \\
\text { Caminhada: } \downarrow 1,6\end{array}$ & $\begin{array}{l}\text { Natação: } \uparrow 1,2 \\
\text { Caminhada: } \downarrow 1,2\end{array}$ & $\begin{array}{l}\text { Nataçăo: } \uparrow 1,2 \\
\text { Caminhada: } s / \\
\text { alteraçăăo }\end{array}$ \\
\hline Simöes et al. ${ }^{(1)}$ & Hidroginástica & 2 & 2 & $45-50$ & \begin{tabular}{|l|}
$\begin{array}{l}\text { Năo há } \\
\text { detalhamento }\end{array}$ \\
\end{tabular} & \begin{tabular}{|l|}
$10 \%$ nẵo completou 0 \\
programa (motivo nẵo \\
referente ao estudo)
\end{tabular} & $\begin{array}{l}\text { Repouso: } \downarrow 1,7 \\
\text { Esforço: } \downarrow 1,6 \\
\end{array}$ & \begin{tabular}{|l|} 
Repouso: $\downarrow 37,7$ \\
Esforço: $\downarrow 15,3$ \\
\end{tabular} & \begin{tabular}{|l|} 
Repouso: $\uparrow 5$ \\
Esforço: $\downarrow 43$ \\
\end{tabular} & \begin{tabular}{|l|} 
Repouso: $\uparrow 3,2$ \\
Esforço: 15,8 \\
\end{tabular} \\
\hline Gimenes et al..$^{(19)}$ & Hidroginástica & 3 & 2 & 45 & $\begin{array}{l}\begin{array}{l}\text { Não há } \\
\text { detalhamento }\end{array} \\
\end{array}$ & \begin{tabular}{l|} 
Năo há detalhamento \\
(nenhum voluntário foi \\
excluído)
\end{tabular} & $\uparrow 1,2$ & $\uparrow 4,2$ & $\begin{array}{l}\text { Não há } \\
\text { detalhamento }\end{array}$ & $\begin{array}{l}\text { Não há } \\
\text { detalhamento }\end{array}$ \\
\hline Piazza et al..$^{(13)}$ & Hidroginástica & 1,75 & 2 & 60 & $\mathrm{FC}_{\text {LR }}$ & $280 \%$ & \begin{tabular}{|l|} 
Sem \\
diferenças \\
(Nấo há \\
detalhamento) \\
\end{tabular} & $\begin{array}{l}\text { Sem diferenças } \\
\text { (Nấo há } \\
\text { detalhamento) }\end{array}$ & $\begin{array}{l}\text { Năo há } \\
\text { detalhamento }\end{array}$ & $\begin{array}{l}\text { Năo há } \\
\text { detalhamento }\end{array}$ \\
\hline Chen et al. ${ }^{(20)}$ & Natação & 12 & $\begin{array}{l}\text { Nẫo há } \\
\text { detalhamen } \\
\text { to }\end{array}$ & \begin{tabular}{|l|} 
Năo há \\
detalhament \\
o
\end{tabular} & $\begin{array}{l}\text { Não há } \\
\text { detalhamento }\end{array}$ & $\begin{array}{l}\text { Não há detalhamento } \\
\text { (nenhum voluntário foi } \\
\text { excluído) }\end{array}$ & $\begin{array}{l}\text { Hipertensos: } \\
\downarrow 11,6 \\
\text { Normotensos: } \\
\uparrow 4,8 \\
\text { (Obs: dado } \\
\text { aproximado, } \\
\text { extraído de } \\
\text { gráfico) } \\
\end{array}$ & $\begin{array}{l}\text { Hipertensos: } 12,5 \\
\text { Normotensos: } 12,8 \\
\text { (Obs: dado } \\
\text { aproximado, } \\
\text { extraído de gráfico) }\end{array}$ & $\begin{array}{l}\text { Não há } \\
\text { detalhamento }\end{array}$ & $\begin{array}{l}\text { Nẫo há } \\
\text { detalhamento }\end{array}$ \\
\hline Farahani et al. ${ }^{(22)}$ & $\begin{array}{l}\text { Hidroginástica + } \\
\text { nataçẳo }\end{array}$ & 2,5 & 3 & 55 & \begin{tabular}{|l|}
$60-65 \%-70-75 \%$ \\
$\mathrm{FC}_{\text {ret }}$ \\
\end{tabular} & $293 \%$ & $\begin{array}{l}\text { Intervençẳo: } \\
\downarrow 10,8 \\
\text { Controle: } \uparrow 1,2 \\
\end{array}$ & \begin{tabular}{|l|} 
Intervençåa: $\uparrow 4,5$ \\
Controle: $\downarrow 0,01$ \\
\end{tabular} & $\begin{array}{l}\text { Não há } \\
\text { detalhamento }\end{array}$ & $\begin{array}{l}\text { Não há } \\
\text { detalhamento }\end{array}$ \\
\hline Nualmin et al. ${ }^{(22)}$ & Nataçẫo & 3 & 3-4 & $15-20$ & $\begin{array}{l}60-70-75 \% \\
\mathrm{FC}_{\text {max }} \\
\end{array}$ & $>99 \%$ & \begin{tabular}{|l|} 
Intervençắo: \\
J6,9 \\
Controle: 0 \\
\end{tabular} & $\begin{array}{l}\text { Intervençẫo: }\rfloor 5,3 \\
\text { Controle: } 11,3 \\
\end{array}$ & $\begin{array}{l}\text { Intervençã̃o: } \mathbf{J 6 , 5} \\
\text { Controle: } 16,3 \\
\end{array}$ & $\begin{array}{l}\text { Não há } \\
\text { detalhamento }\end{array}$ \\
\hline Bündchen $^{(12)}$ & $\begin{array}{l}\text { Caminhad a/corrid } \\
\text { a +força de } \\
\text { MMSS'MMII }\end{array}$ & 6 & 3 & \begin{tabular}{|l|} 
Aeróbio: 30 \\
Força: 2 \\
séries de 12 \\
repetiçőes \\
Total: nẫo \\
há \\
detalhament \\
n
\end{tabular} & 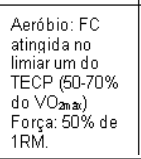 & Nẵo há detalhamento & $\uparrow 0,29$ & $\uparrow 0,4$ & $\begin{array}{l}\text { Nẫo há } \\
\text { detalhamento }\end{array}$ & $\begin{array}{l}\text { Nẫo há } \\
\text { detalhamento }\end{array}$ \\
\hline $\begin{array}{l}\text { Guimarães et } \\
\text { al. }{ }^{\left({ }^{(26)}\right.}\end{array}$ & Hidroginástica & 9 & 3 & 60 & $\begin{array}{l}11-13 \text { escala de } \\
\text { Borg }\end{array}$ & $100 \%$ & \begin{tabular}{|l|} 
Intervençäo: \\
J12,4 \\
Controle: $\uparrow$ \\
(nấo há \\
detalhamento) \\
\end{tabular} & $\begin{array}{l}\text { Intervençẫo: } \backslash 111,1 \\
\text { Controle: } \uparrow \text { (nấo há } \\
\text { detalhamento) }\end{array}$ & $\begin{array}{l}\text { Intervenção: } 15,3 \\
\text { Controle: } \uparrow 2,4\end{array}$ & $\begin{array}{l}\text { Nẫo há } \\
\text { detalhamento }\end{array}$ \\
\hline $\begin{array}{l}\text { Santos Costa } \\
\text { Cruel }^{(23)}\end{array}$ & $\begin{array}{l}\text { Exercícios } \\
\text { aeróbicos } \\
\text { aquáticos } \\
\text { (hidroginástica, } \\
\text { natacáano e corrida } \\
\text { aquática) }\end{array}$ & 0,5 a 12 & 2 & \begin{tabular}{|l|} 
Aquático: \\
$20-60$ \\
(média 55) \\
Aeróbio \\
solo: 20-50 \\
(média 31) \\
\end{tabular} & $\begin{array}{l}\text { Entre moderada } \\
\text { e alta (nåo há } \\
\text { detalhamento) }\end{array}$ & Não há detalhamento & $\begin{array}{l}\downarrow \text { sem } \\
\text { detalhamento }\end{array}$ & $\downarrow$ sem detalhamento & $\begin{array}{l}\text { Não há } \\
\text { detalhamento }\end{array}$ & $\begin{array}{l}\text { Não há } \\
\text { detalhamento }\end{array}$ \\
\hline Zaar $^{(25)}$ & \begin{tabular}{l|} 
Determinados de \\
acordo com a \\
condiçăo de \\
saúde individual, \\
idade, aptiazáo \\
física, etc... (năo \\
há detalhamento)
\end{tabular} & 24 & 3 & 60 & $\begin{array}{l}50 \% \text { a } 80 \% \\
\mathrm{FC}_{\text {suthmacma }}\end{array}$ & Năo há detalhamento & $\begin{array}{l}\text { Normotensos: } \\
11,6 \\
\text { pre- } \\
\text { hipertensos: } \\
\mathbb{} 6,7\end{array}$ & $\begin{array}{l}\text { Normotensos: } 16,8 \\
\text { pré-hipertensos: } \\
13,8\end{array}$ & $\begin{array}{l}\text { Năo há } \\
\text { detalhamento }\end{array}$ & $\begin{array}{l}\text { Não há } \\
\text { detalhamento }\end{array}$ \\
\hline Felicio et al. ${ }^{(27)}$ & 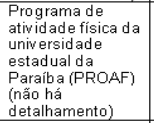 & 9 & Sem dados & \begin{tabular}{|l|} 
Nẫo há \\
detalhament \\
o
\end{tabular} & $\begin{array}{l}\text { Nẫo há } \\
\text { detalhamento }\end{array}$ & Năo há detalhamento & $\$ 13,2$ & \rfloor 15,3 & $\begin{array}{l}\text { Não há } \\
\text { detalhamento }\end{array}$ & $\begin{array}{l}\text { Nẫo há } \\
\text { detalhamento }\end{array}$ \\
\hline Miranda et al. ${ }^{(23)}$ & $\begin{array}{l}\text { Caminhada + } \\
\text { exercícios } \\
\text { calistênicos }+ \\
\text { exercícios com } \\
\text { materiais } \\
\text { alternativos }+ \\
\text { academia da } \\
\text { saúde }\end{array}$ & 3,25 & 3 & 60 & $\begin{array}{l}\text { Não há } \\
\text { detalhamento }\end{array}$ & Não há detalhamento & $\begin{array}{l}\text { Não há } \\
\text { detalhamento }\end{array}$ & $\begin{array}{l}\text { Não há } \\
\text { detalhamento }\end{array}$ & $\begin{array}{l}\text { Não há } \\
\text { detalhamento }\end{array}$ & $\begin{array}{l}\text { Não há } \\
\text { detalhamento }\end{array}$ \\
\hline Hortencio $^{(22)}$ & $\begin{array}{l}\text { Caminhada inicial } \\
\text { + exercicios livres } \\
\text { em circuito } \\
\text { (MMSS/tonco) + } \\
\text { caminhada final }\end{array}$ & 3 & 2 & 90 & $\begin{array}{l}\text { Leve a } \\
\text { moderada (năo } \\
\text { há } \\
\text { detalhamento) }\end{array}$ & $\begin{array}{l}\text { Permitida até } 25 \% \text { de } \\
\text { faltas (37,03\% foram } \\
\text { excluidos) }\end{array}$ & $\$ 13,7$ & \rfloor 16,3 & $\begin{array}{l}\text { Não há } \\
\text { detalhamento }\end{array}$ & $\begin{array}{l}\text { Não há } \\
\text { detalhamento }\end{array}$ \\
\hline Damásio et al ${ }^{[30]}$ & \begin{tabular}{l|} 
Exercícios \\
dinâmicose \\
ativos de \\
MMSS/MMII (em \\
equipamentos de \\
musculaçâo) \\
esteira/bicicleta \\
\end{tabular} & 6 & 3 & 50 & $\begin{array}{l}\text { Moderada (60- } \\
70 \% \text { VO=2nax) }\end{array}$ & $\begin{array}{l}\text { Voluntários que nẫo } \\
\text { atingiram frequência } \\
\text { mínima de participaçâa } \\
\text { foram excluídos (Nâao } \\
\text { há detalhamento) }\end{array}$ & $\downarrow 13,4$ & $\begin{array}{l}\text { Não há } \\
\text { detalhamento }\end{array}$ & $\$ 22,2$ & $\begin{array}{l}131,2 \\
\text { (Ob's: dado } \\
\text { aproximado, } \\
\text { extraído de gráfico) }\end{array}$ \\
\hline $\operatorname{Lima}^{(31)}$ & $\begin{array}{l}\text { Cinesioterapia de } \\
\text { forma ativa } \\
\text { (tratamento } \\
\text { fisioterápico) }\end{array}$ & 1,5 & Semdados & \begin{tabular}{|l|}
$\begin{array}{l}\text { Não há } \\
\text { detalhament } \\
\text { o }\end{array}$ \\
\end{tabular} & $\begin{array}{l}\text { Escala de Borg } \\
\text { e FC rep (năo hă } \\
\text { detalhamento) }\end{array}$ & $\begin{array}{l}11 \text { voluntários foram } \\
\text { excluídos devido às } \\
\text { faltas (52,4\%) (Nấo há } \\
\text { detalhamento) }\end{array}$ & $\$ 6,5$ & $\mid \downarrow 16,6$ & $\downarrow 2,7$ & 17,8 \\
\hline Cassiano et al[. & $\begin{array}{l}\text { Exercícios } \\
\text { funcionais em } \\
\text { circutito } \\
\text { caminhada }\end{array}$ & 4 & $\begin{array}{l}2 \\
\text { (Exercícios } \\
\text { funcionais) } \\
1 \\
\text { rCaminhad } \\
\text { a) }\end{array}$ & 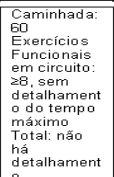 & 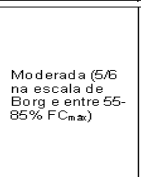 & $\begin{array}{l}\text { Ev voluntário s näo } \\
\text { finalizaram o programa } \\
(11.1 \%)\end{array}$ & 16,0 & $\downarrow 5,4$ & 12,9 & $+3,3$ \\
\hline
\end{tabular}


A Figura 1 retrata uma dispersão entre o volume de treinamento e a porcentagem de alteração na PAS e PAD dos estudos provenientes da literatura (detalhados no Quadro 2) que disponibilizaram essas informações estão apresentadas na Figura 1. Observou-se correlação moderada do volume de treinamento com a redução percentual da PAS $(r=0,53 ; p<0,05)$ e baixa com a redução percentual da PAD $(\mathrm{r}=0,33 ; \mathrm{p}<0,05)$.
Não foi possível ajustar a alteração percentual da PAS e PAD pela intensidade dos exercícios devido a ampla diversidade de ferramentas utilizadas em cada estudo, e nem pela frequência de participação dos voluntários, uma vez que a maioria dos estudos não trouxe essas informações. Além disso, poucos estudos trouxeram informações sobre a frequência cardíaca de repouso e o duplo produto, o que nos impediu de verificar as associações dessas

\section{Figura 1. Dispersão entre o volume de treinamento e a porcentagem (\%) de alteração na Pressão Arterial Sistólica (PAS) e Diastólica (PAD).}

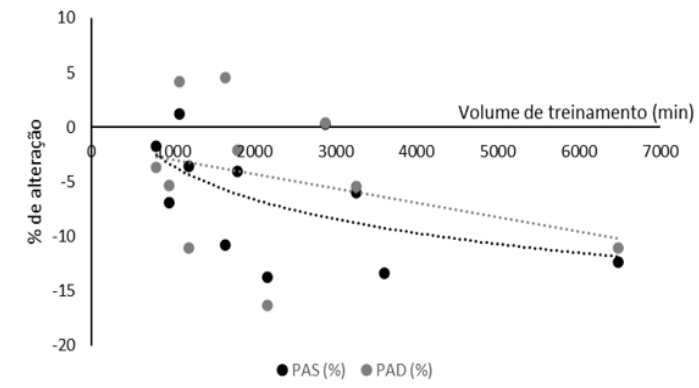

Nota: dados provenientes de estudos da literatura (detalhados no Quadro II).

variáveis com o volume de treinamento.

Portanto, independentemente do tipo de exercício praticado, a assiduidade e o volume de treinamento realizado são variáveis influentes na magnitude das reduções de indicadores de hipertensão arterial.

\section{CONCLUSÃO}

O exercício físico tem papel essencial no tratamento não medicamentoso para controle da hipertensão arterial e na manutenção da qualidade de vida dos indivíduos. Após o início da prática do exercício físico surgem fatores motivacionais que vão desde perda de medidas corporais, redução do estresse e até economia em razão da diminuição do uso de fármacos. O volume de treinamento físico realizado tem influência sobre a magnitude de redução dos indicadores relacionados à hipertensão arterial sistêmica. Portanto, é preciso investir em políticas públicas e projetos de exercício físico nas unidades básicas de saúde dos municípios para divulgar o exercício físico como agente fundamental de benefícios para os usuários que possuem essa comorbidade. 
A prática regular de exercício físico no controle da hipertensão arterial

\section{REFERÊNCIAS}

1. Morais SRd, Bezerra IN, Souza AdM, Vergara CMAC, Sichieri R. Alimentação fora de casa e biomarcadores de doenças crônicas em adolescentes brasileiro. Cadernos de Saúde Pública. 2021;37(1):1-14.

2. Carvalho AS, Abdalla PP, Júnior JRG, Venturini ACR, Vilela Junior GB. Atividade física e seus diferentes métodos de análise: uma revisão narrativa. Centro de pesquisa avançada em qualidade de vida CPAQV. 2021;13(1):1-11.

3. Castilho I. Hipertensão é diagnosticada em 24,7\% da população, segundo a pesquisa Vigitel: <http:/ / www.saude.gov.br/noticias/agencia-saude/45446-nobrasil-388-pessoas-morrem-por-dia-por-hipertensao $>$. ; 2019 [cited 2019].

4. Souza CP, Valentim MCP, Ferreira AD, Abdalla PP, Silva LSL, Carvalho AS, et al. Prevalência de doenças crônicas não transmissíveis, hábitos alimentares e de atividade física numa estratégia de saúde da família de presidente prudente - SP. Conscientiae Saúde. 2020;19(1):1-15.

5. Krummel D. Terapia Nutricional na Hipertensão In: Mahan LK, Escott-Stump S. Krause: Alimentos, Nutrição e Dietoterapia. . Campinas: Papirus; 2005.

6. Carvalho AS, Abdalla PP, Bueno Júnior CR. Atuação do profissional de educação física no sistema único de saúde: revisão sistemática. Revista Brasileira em Promoção da Saúde. 2017;30(3):1-11.

$7 . \quad$ Veloza L, Jiméneza C, Quiñonesa D, Polanía F, Pachón-Valero LC, Rodríguez-Triviño CY. Variabilidad de la frecuencia cardiaca como factor predictor de las enfermedades cardiovascularesHeart rate variability as a predictive factor of cardiovascular diseases. Revista Colombiana de Cardiología. 2019;26(4):205-10.

8. Carvalho AS, Alves TC, Abdalla PP, Venturini ACR, Leites PDL, Machado DRL. Composição corporal funcional: breve revisão. Caderno de Educação Física e Esporte. 2018;16(1):1-12.

9. Matavelli IS, Judice ELD, Matavelli R, Hunger MS, Martelli A. Hipertensão Arterial Sistêmica e a Prática Regular de Exercícios Físicos como Forma de Controle: Revisão de Literatura. Revista Brasileira de Ciências da Saúde. 2014;18(4):359-66.

10. Cruz MRA, Lima ENS, Santos NVP, Linhares NP, Lima AGT. O papel das intervenções não farmacológicas para controle da hipertensão arterial: revisão integrativa. Brazilian Journal of Development. 2021;7(3):29330-44.

11. Matos JS. Perceção de efeitos adversos por doentes hipertensos e sua relação com não adesão voluntária à terapêutica. Coimbra Portugal: Universidade de Coimbra; 2020.

12. Bündchen DC, Schenkel IdC, Santos RZd, Carvalho Td. Exercício físico controla pressão arterial e melhora qualidade de vida. Revista Brasileira de Medicina do Esporte. 2013;19(2):91-5.

13. Tanaka H, Junior DRB, Howley ET, Thompson DL, Ashraf M, Rawson FL. Swimming training lowers the resting blood pressure in individuals with hypertension. Journal of Hypertension. 1997;15(6):651-7.

14. Arca EA, Fiorelli A, Rodrigues AC. Efeitos da hidrocinesioterapia na pressão arterial e nas medidas antropométricas em mulheres hipertensas. Revsita Brasileira de Fisioterapia. 2004;8(3):279-83.

15. Ciolac EG, Guimarães GV. Exercício físico e síndrome metabólica. Revista Brasileira Medicina do Esporte. 2004;10(4):319-24.

16. Cox KL, Burke V, Beilin JL, Grove RJ, Blanksby BA, Puddey IB. Blood pressure rise with swimming versus walking in older women: the Sedentary Women Exercise Adherence Trial 2 (SWEAT 2). Journal of Hypertension. 2006;24(2):307-14.

17. Simões RA, Horii L, Carraro R, Simões R, Cesar MdC, Montebello MIdL. Efeitos do treinamento de hidroginástica na aptidão cardiorrespiratória e nas variáveis hemodinâmicas de mulheres hipertensas. Revista Brasileira de Atividade Física \& Saúde. 2007;12(1):1-7.

18. Piazza L, Menta MR, Castoldi C, Reolão JBC, Schmidt R, Calegari L. Efeitos de exercícios aquáticos sobre a aptidão cardiorrespiratória e a pressão arterial em hipertensas. Fisioterapia e Pesquisa. 2008;15(3):28591.

19. Gimenes RO, Carvalho NTPd, Farelli BC, Mello TWPd. Impacto da Fisioterapia Aquática na Pressão arterial de Idosos. O Mundo da Saúde São Paulo. 2008;32(2):170-5.

20. Chen H-h, Chen Y-L, Huang C-Y, Lee S, Chen $\mathrm{S}$, Kuo C-H. Effects of one-year swimming training on blood pressure and insulin sensitivity in mild hypertensive young patients. The Chinese journal of physiology. 2010;53(3):185-9.

21. Guedes NG, Lopes MVdO. Exercício físico em portadores de hipertensão arterial: uma análise conceitual. Revista Gaucha de Enfermagem. 2010;31(2):367-74.

22. Farahani AV, Mansournia M-A, Asheri $H$, Fotouhi A, Yunesian M, Jamali M, et al. The effects of a 10-week water aerobic exercise on the resting blood pressure in patients with essential hypertension. Asian Journal of Sports Medicine 2010;1(3):159-67.

23. Nualnim N, Parkhurst K, Dhindsa M, TakashiTarumi, Vavrek J, Tanaka H. Effects of swimming training on blood pressure and vascular function in adults $>50$ years of age. The American Journal of Cardiology. 2012;109(7):1005-10.

24. Santos NS, Costa RFd, Kruel LFM. Efeitos de exercícios aeróbicos aquáticos sobre a pressão arterial em adultos hipertensos: revisão sistemática. Revista Brasileira de Atividade Física \& Saúde. 2014;19(5):54858.

25. Zaar A, Reis VM, Sbardelotto ML. Efeitos de um programa de exercícios físicos sobre a pressão arterial e medidas antropométricas. Revista Brasileira de Medicina do Esporte. 2014;20(1):13-6.

26. Guimaraes GV, Cruz LGdB, Fernandes-Silva MM, Dorea EL, Bocchi EA. Heated water-based exercise training reduces 24-hour ambulatory blood pressure levels in resistent hypertensive patients: A randomized controlled trial (HEx trial). International Journal of Cardiology. 2014;172(2):434-41.

27. Felício IM, Diniz AFA, Junior EBA, Dantas DS, Barros DdS, Batista PdN, et al. Atividade física no equilíbrio metabólico da hipertensão arterialBiofarm. 2017;13(2):39-43.

28. Miranda ÉÁ, Ferreira AM, Montserrat PM, Oliveira GHMd. Efeitos de um programa de exercício físico ao ar livre em diabéticos e hipertensos atendidos 
em um núcleo de apoio à saúde da família de lagoa da prata-MG. Revista Brasileira de Prescrição e Fisiologia do Exercício. 2017;11(65):164-72.

29. Hortencio MNdS, Silva JKS, Zonta MA, Melo CPAd, França CN. Efeitos de exercícios físicos sobre fatores de risco cardiovascular em idosos hipertensos. Revista Brasileira em Promoção da Saúde. 2018;31(2):19.

30. Damázio LCM, Filho GRdS, Pereira ACdL, Leão TLdO, Ferreira LC, Mourão FN. Os efeitos de um programa de atividade física na pressão arterial e frequência cardíaca de pacientes hipertensos e deficientes físicos. Revista de Atenção à Saúde. 2019;17(59):81-7.

31. Lima MF. Análise dos efeitos do exercício físico aplicado aos pacientes com hipertensão arterial sistêmica e diabetes de mellitus assistidos pela equipe de estratégia saúde e família da cidade de Paracatu-MG. Humanidades \& tecnologia. 2019;16(1):474-93.

32. Cassiano AdN, Silva TSd, Nascimento CQd, Wanderley EM, Prado ES, Santos TMdM, et al. Efeitos do exercício físico sobre o risco cardiovascular e qualidade de vida em idosos hipertensos. Ciência \& Saúde Coletiva. 2020;25(6):2203-12.

33. Vargas EVM, - FCC, Pinto VS, Gomes DV. Efeito agudo dos exercícios resistidos e exercícios aeróbicos sobre a pressão arterial de homens hipertensos leves. Ponteditora. 2020;2(1):57-70.

34. Deus LA, Simões HG, Neves RVP, Souza MK, Moraes MR, Navarro F, et al. Associação entre a variabilidade da frequência cardíaca e o estresse oxidativo: o papel do exercício. Revista Brasileira De Prescrição E Fisiologia Do Exercício. 2017;11(66):36676.

OBSERVAÇÃO: Os autores declaram não existir conflitos de interesse de qualquer natureza. 